\title{
30Gb/s absolute polar duty cycle division multiplexing in dispersion uncompensated optical systems
}

\begin{abstract}
In this study the author has modeled and characterizes the performance of 3times $10 \mathrm{Gbp} / \mathrm{s}$ absolute polar duty cycle division (APDCDM) multiplexing in dispersive environments at $1550 \mathrm{~nm}$. APDCDM technique is examined, with comparison to non-return-to-zero (NRZ) and return-to-zero (RZ) time division multiplexing. In this paper three channel operating at the same speed of $10 \mathrm{Gbps}$ are multiplexed in electrical domain. The experimental simulation results show that the receiver sensitivity of all users in APDCDM system is similar to that of RZ-TDM and $3 \mathrm{~dB}$ better than NRZ-TDM. The proposed system offer reduced dispersion sensitivity; this suggests advantages for APDCDM in optical multiplexing systems. It was also showed that APDCDM can support higher bit rate than TDM and also, it is less sensitive to the chromatic dispersion effect.
\end{abstract}

Keyword: Optical communication; Multiplexing technique; Dispersion uncompensat; Highspeed network 